\title{
Load Balanced Fuzzy Control based Adaptive Gateway Discovery for Ubiquitous Internet Access in MANET
}

\author{
GARGI BHARDWAJ1, UDAI SHANKAR2 \\ Department of Computer science \& Engineering and Information Technology \\ Uttar Pradesh Technical University, Lucknow-India
}

\begin{abstract}
Integrating MANETs (Mobile Ad-hoc Networks) nodes to the Internet require either a connection to the Internet or they can connect to the Internet through the Internet gateways. For the second case if a node in a MANET has discover the gateway to connect to the fixed hosts in the Internet and it can be done either by broadcasting a gateway discovery request message initiated by nodes itself or by broadcasting periodic gateway advertisement messages from the gateways. End to end packet delay and throughput are strongly dependent on the time needed to discover the gateways.Nodes in a MANET use number of hops as metric to select a path to a gateway, if all the nodes select its nearest gateway to access the Internet then there may be situations when the network performance degrades because some nodes along the path have too many packets waiting in the queue. This paper presents a novel solution to access internet through mobile nodes in ad hoc network. Here the gateway replies with an advertisement message which is broadcasted to the whole network instead of sending a unicast reply to the requesting node. The traffic load taken into account along a path in addition with minimum hop count to select an efficient gateway. The AODV routing protocol has been used for routing in the MANET domain.A new strategy of gateway discovery was investigated and impact of new metric on the gateway selection in NS-2 was observed. Simulation results show that the proposed scheme outperforms over the existing scheme with high throughput and lower end to end delay.
\end{abstract}

KEYWORDS: MANET; AODV; Internet Gateway Discovery; Internet; Routing Protocols; Mobile IP; Address Autoconfiguration.

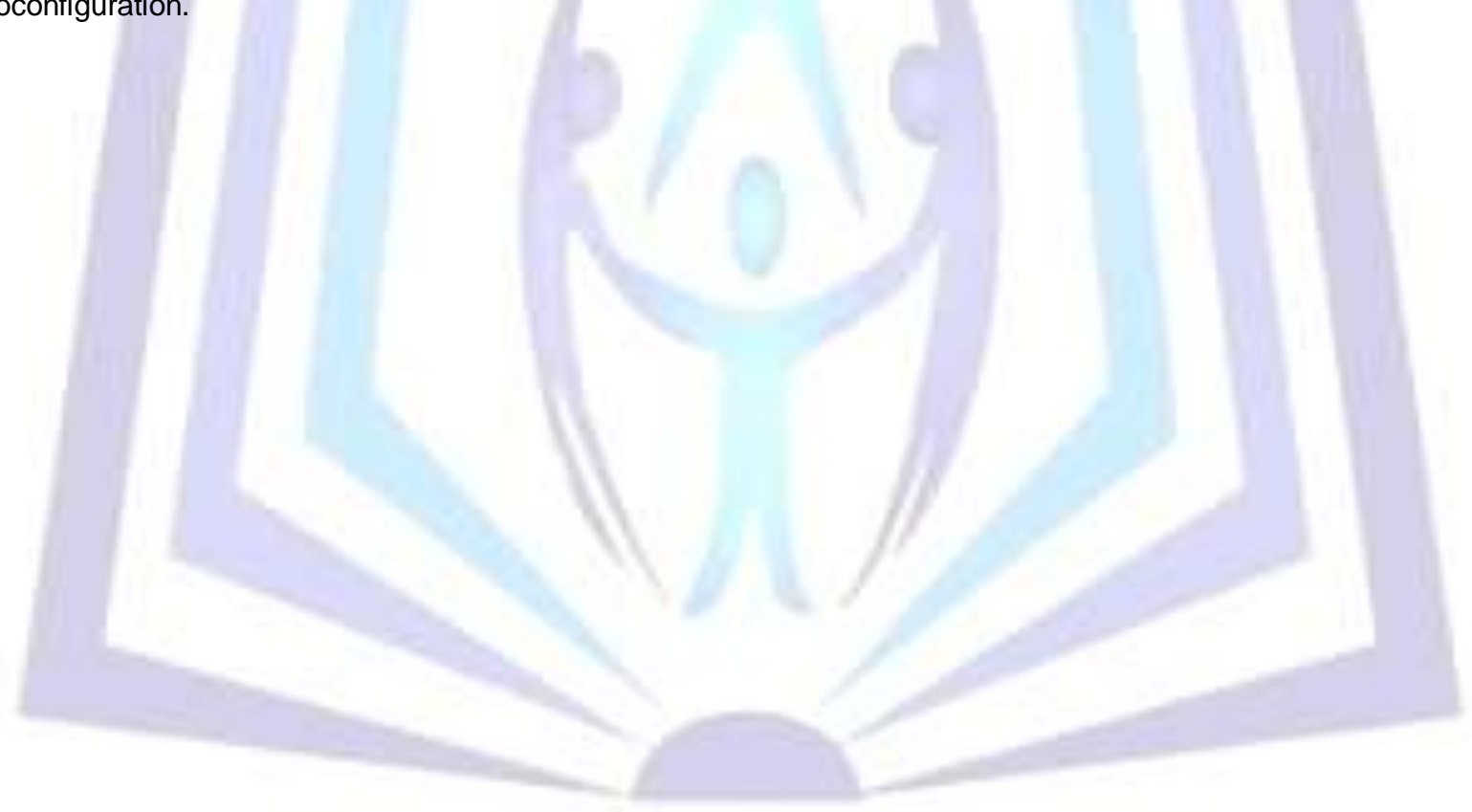

\section{Council for Innovative Research}

Peer Review Research Publishing System

\section{Journal: INTERNATIONAL JOURNAL OF COMPUTERS \& TECHNOLOGY}

Vol. 14, No. 12

www.ijctonline.com, editorijctonline@gmail.com 


\section{INTRODUCTION TO MANET AND INTERNET}

MANET-Internet integration [1] is a heterogeneous network which interconnects wired Internet to the wireless (MANET). This interconnection is achieved using Internet gateways which act as access point between these two different types of networks and help to exchange packets between MANET node and internet host. In order to extend the benefit of a MANET, it is interconnected to the Internet which lets mobile devices within the MANET to communicate with wired nodes in the Internet and to access services provided by the Internet. This interconnectivity results in a heterogeneous wiredcum-wireless network which has been referred in the literature by various terms like infrastructure based MANET or Internet Integrated MANET (IIM) [1]. This wired-cum wireless network differs from solutions like Mobile IP [2], since Mobile IP offers one-hop internet connectivity whereas IIM offers multi-hop connectivity. Architecture of heterogeneous wiredcum-wireless network is shown in fig1 and various issues in MANET-Internet integration are discussed in [3].

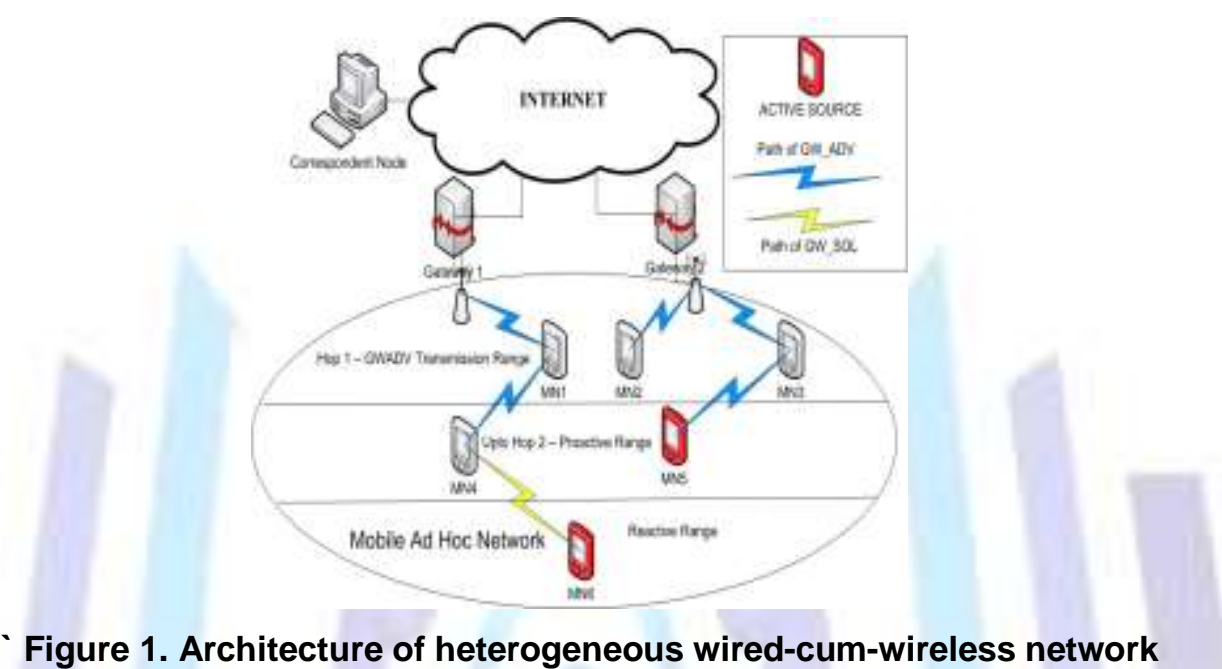

Some of the important issues in this interconnection includes: seamless routing of packets from one type of network to the other, location management of mobile nodes, single point of failure regarding gateways, gateway load balancing, addressing, and efficient discovery of gateways by mobile nodes in the MANET. The issues of gateway load balancing are addressed in [4-7] and also efficient adaptive gateway discovery are discussed [8-11]. But none of the authors have addressed these two issues together. These two issues are closely related since gateway load balancing entails adjusting the gateway discovery parameters. In this paper, the two issues of gateway load balancing and gateway discovery are addressed together. Gateway load balancing adjusts the load on the Internet gateway using a gateway load balancing mechanism. Gateway discovery mechanisms adjust the gateway advertisement periodicity and TTL value. In this paper, the WLB-AODV (Weighted Load Balanced-AODV) [7] routing protocol is used for gateway load balancing in IIM. A Fuzzy logic based control mechanism is proposed based on the Mamdani fuzzy control architecture that dynamically adjusts the GW_ADV periodicity based on three parameters namely: Aggregate Interface Queue Length (AIQL), Average Hops (AH), and Number of nodes registered with the gateway $(\mathrm{NsN})$. The issues of adjusting the TTL value dynamically are not addressed in this paper.

The rest of the paper is organized as follows: section 2 presents work related work to the proposed mechanism, while the proposed work is presented in section 3 . The section 4 presents simulation setup and work analysis and finally section 5 concludes the paper with future directions.

\section{RELATED WORK}

A modified form of the AODV routing protocol called WLB-AODV is proposed in [7] which integrated gateway load balancing into the routing protocol. A review of gateway load balancing strategies is performed in [12]. A fuzzy control mechanism was used in [13] in order to adjust the GW_ADV periodicity dynamically. None of the above gateway load balancing strategies implements adaptive gateway discovery mechanisms .Zaman et al proposed Fuzzy Control based adaptive gateway discovery for Internet access in MANET" stating that the Gateway discovery and gateway load balancing are important issues in Integrated Internet-MANET and have been addressed separately in the literature. In this paper a new solution which addresses these two issues together is proposed. In the proposed mechanism, the problem of finding efficient values to gateway discovery parameters is treated as a control system which uses the Mamdani Architecture for Fuzzy Control. Gateway load balancing is achieved through the WLB-AODV routing protocol. The proposed protocol has been simulated in ns-2 network simulator. Simulation results show that the proposed protocol gives better packet delivery ratio and comparable end to end delay to the existing solution [14]. 
Zaman et al in their paper entitled "Load balanced fuzzy control based adaptive gateway discovery mechanism. In this paper, the issues of gateway load balancing and gateway discovery are addressed together. The proposed mechanism uses the WLB-AODV routing protocol for gateway load balancing and treats the problem of finding efficient values to gateway discovery parameters as a control system and uses the Mamdani Architecture for Fuzzy Control.

[15].

Yonghang et. al. proposed "QoS-based gateway selection in MANET with Internet connectivity" based on three QoS metrics: traffic load of gateway, path quality from MANET node to the gateway and hop count to the gateway for integrating MANET and the Internet. The Simple Additive Weighting method is used to combine these three QoS metrics to outrank the optimum gateway. Gateway with the smallest weight will be selected as a gateway. Simulation results show that the proposed scheme can improve packet delivery ratio and end to end delay [16].

\section{PROPOSED WORK:}

\section{GATEWAY DISCOVERY MECHANISNM PROTOCOL:}

The protocol has two main components that are, Gateway load balancing and adaptive gateway discovery. Gateway load balancing is achieved using WLB-AODV routing protocol. Adaptive gateway discovery is divided into two parts, adjusting the TTL value of the GW_ADV and adjusting the periodicity of the TTL value. The maximal source coverage mechanism is used to adjust the TTL value. A fuzzy logic control mechanism based on the mamdani architecture is used to dynamically adjust the GW_ADV periodicity. The working procedure of the proposed scheme is presented in the following subsections.

A. WLB-AODV Routing Protocol : This routing protocol was proposed in Gateway load balancing based on the following parameters: aggregate routing table entries (ARTE), hop count (HC), and aggregate interface queue length $(A I Q L)$. The route selection decision is based on the metric $\mathrm{Ri}$ which is computed as per the equation given below:

$\mathrm{Ri}=w 1^{*} \mathrm{HC}+\mathrm{w} 2^{*} \mathrm{AIQL}+\mathrm{w} 3^{*} \mathrm{ARTE}$

Where w1,w2, and w3 are the weights given to the respective parameters HC, AIQL and ARTE. The basic concept of using a load balanced routing protocol in an IIM is to avoid using heavily loaded paths and using lightly loaded paths for transmitting data. In fig 2 the path from MN3 to gateway1 via MN1 is 2 hops long, but highly loaded.

MN3 has another path to gateway1: MN3- MN5-MN2, which is longer but lesser, loaded. WLB-AODV basically uses lesser loaded paths irrespective of their length.

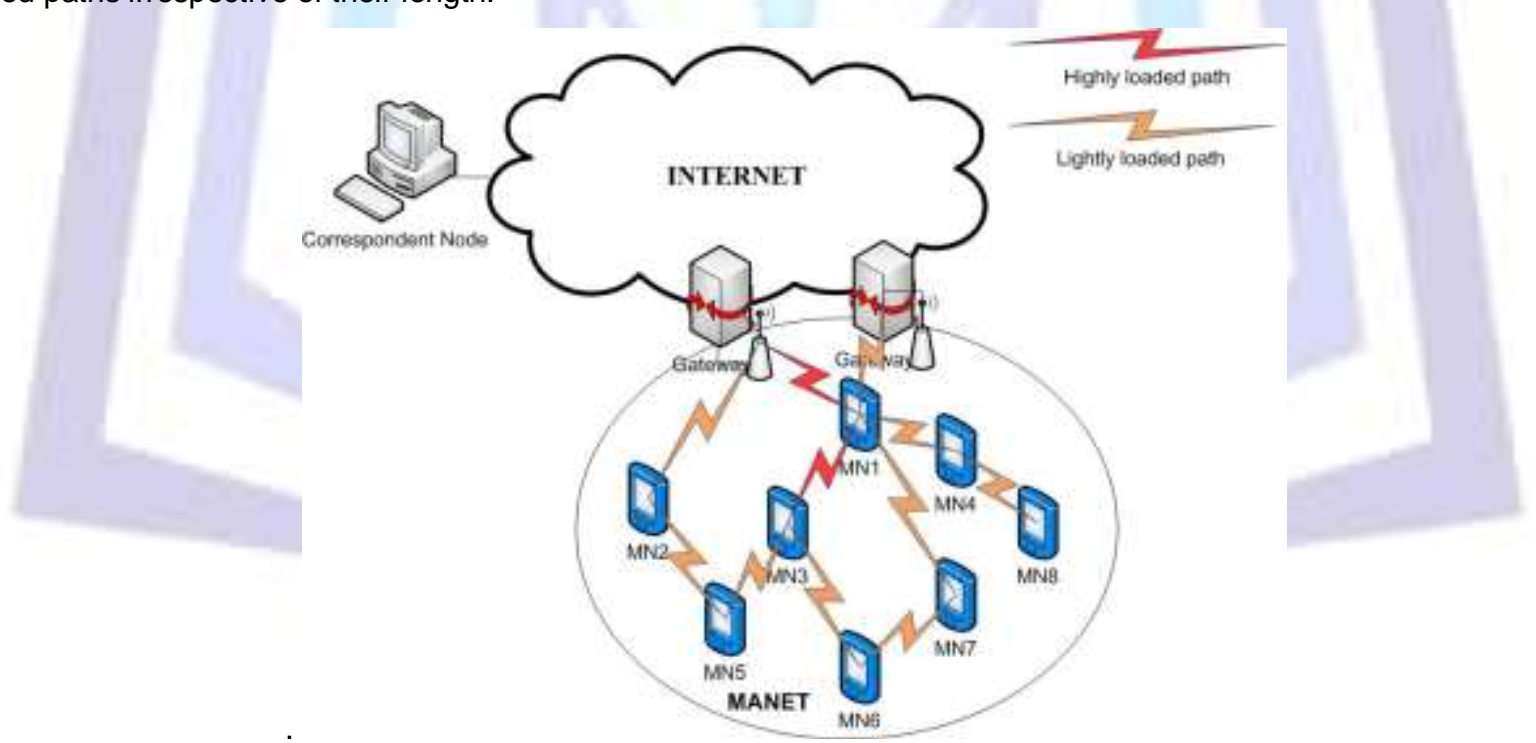

Figure 2. Working of WLB-AODV in MANET-INTERNET Integration

B. Mamdani Fuzzy Control Architecture for adaptive gateway discovery: The Mamdani Fuzzy Control Architecture uses fuzzy rules to control the behavior of a physical system. It basically consists of an inference engine and a rule base. The inference engine is programmed using simple if-then type of rules. The rule base may be built by exploring the knowledge of a subject expert. The physical system is observed for output values (OV). These output values are then fed into the fuzzy control system which uses inference engine and the rule base to produce updated input values which are then fed to the physical system under observation.

C. Adjusting the TTL value: The TTL value of the GW_ADV message is adjusted according to the Maximal Source Coverage algorithm. The maximal source coverage algorithm is an adaptive gateway discovery scheme which focuses on the dynamic adjustment of the TTL value. Active source of a gateway is defined as a mobile node which uses the gateway for Internet connectivity. The TTL value of the gateway advertisement messages in the next cycle is set to the 
distance in hops of the farthest active source of the gateway. The working of the maximal source coverage algorithm is explained using fig 3 . In this figure, initially the proactive range is set to two hops. The active sources which are outside the proactive range, i.e., the source two hops away from the gateway are said to be in the reactive range. MN4 is the active source in the current advertisement cycle which falls outside the proactive range. Therefore, in the next gateway advertisement cycle, the TTL value is set so that it includes MN4 into the proactive range.

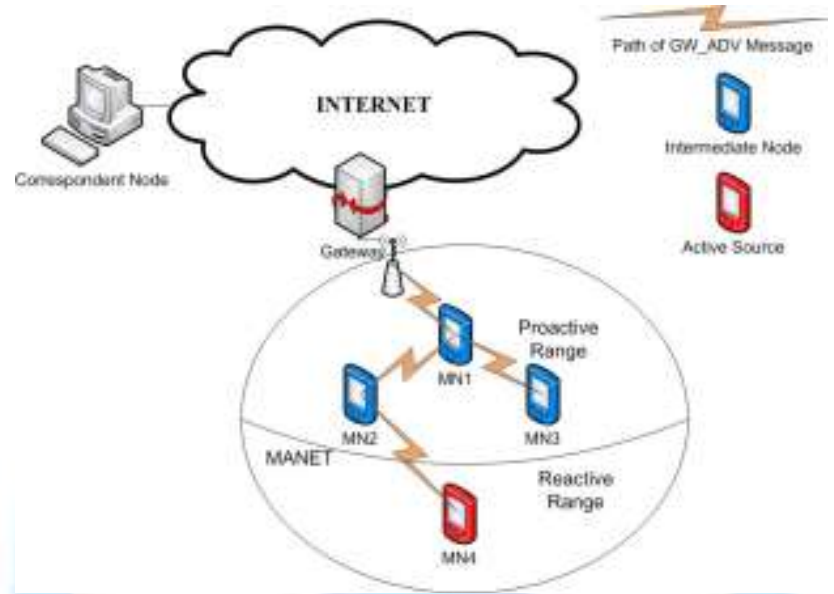

Figure 3. Working of Maximal Source Coverage Algorithm.

D. Adjusting the GW_ADV periodicity: The proposed scheme incorporates the features of adaptive gateway discovery and gateway load balancing. In the proposed scheme the WLB-AODV routing is used to efficiently forward packets within the MANET, hence implementing the gateway load balancing scheme in the proposed protocol. To adjust the periodicity of GW_ADV messages following metrics are used.

- Number of nodes registered with a gateway (NN): It is the number of mobile nodes registered with a gateway to access Internet connectivity.

- Average Hop: The average of the hop counts of all the active sources which use this gateway for Internet connectivity.

- Aggregate Interface Queue Length (AIQL): The number of packets queued in the Interface queue of a gateway. As shown in fig 4, the IIM is the physical system. The three metrics AH, NN and AIQL are the outputs generated by the physical system. These outputs become inputs to the Inference engine. The Inference engine refers the rule base to calculate the periodicity value to be set for the next GW_ADV message cycle. These newly calculated values are given as inputs to the physical system.

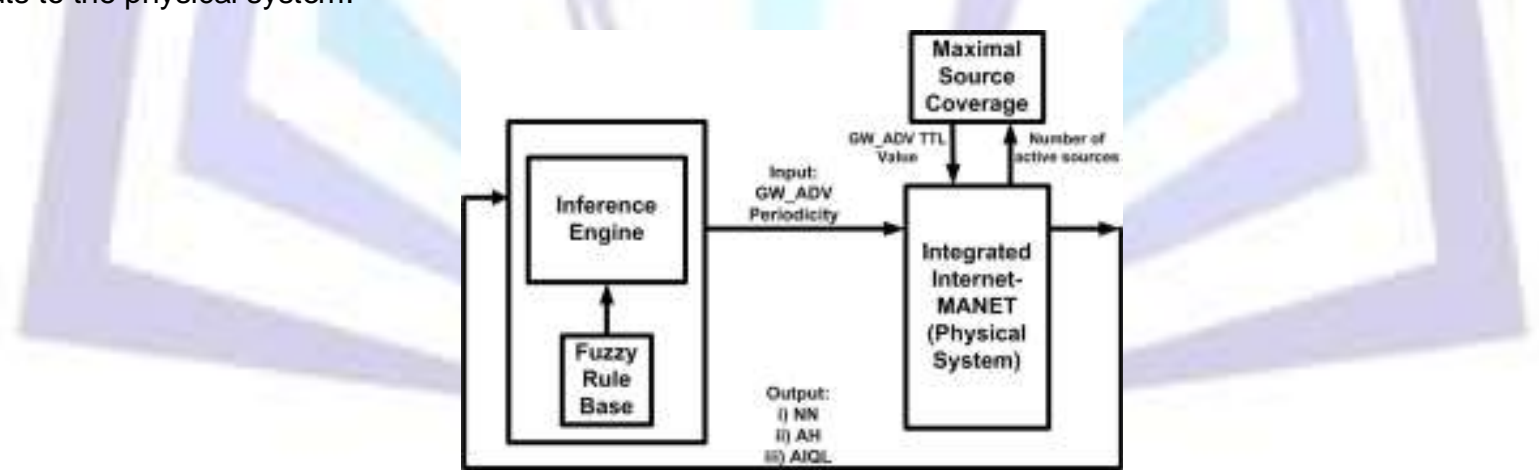

Figure 4. The Mamdani Fuzzy Control Architecture for the Proposed Protocol

To adjust the periodicity of GW_ADV messages, three metrics AH, NN and AIQL are used. The triangular membership function is used to map the values of the three metrics into interval $[0,1]$. The $a, b$ and $c$ values of the triangular functions of the three metrics are presented in the following table 1 :

Table 1. Triangular membership function values

\begin{tabular}{|c|c|c|c|}
\hline Metric & A & b & c \\
\hline Average Hops (AH) & 0 & 1 & 2 \\
\hline Number of Nodes Registered (NN) & 0 & 2 & 4 \\
\hline $\begin{array}{c}\text { Aggregate Interface Queue Length } \\
\text { (AIQL) }\end{array}$ & 250 & 750 & 1500 \\
\hline
\end{tabular}


Let $\mathrm{AHt}, \mathrm{NNt}$, and AIQLt denote the values which are returned by the triangular membership function for the three parameters $\mathrm{AH}, \mathrm{NN}$ and $\mathrm{AIQL}$ respectively. Once the metrics have been mapped into the [0,1] interval, we use the union operator to determine the Al_Need value that is used as the parameter to determine the advertisement interval. The union operator is represented as:

Al_Need $=$ Union $(\mathrm{AHt}, \mathrm{NNt}, \mathrm{AIQLt})$

A high Al_Need value indicates less need for GW_ADV messages and a low value indicates that more GW_ADV messages are required therefore, the periodicity of GW_ADV has to be increased by decreasing the advertisement interval. The Al_Metric value is used as input to the rule base which finally determines the Advertisement Interval (Al) value. The rule base is based on the mamdani fuzzy rules and is presented in Table 2.

Table 2. Fuzzy rule base for determining advertisment interval

\begin{tabular}{|c|c|}
\hline Al_Need Value & $\begin{array}{c}\text { GW_ADV Advertisement } \\
\text { Interval (Secs) }\end{array}$ \\
\hline$>0.8$ & 9 \\
\hline$<=0.8$ and $>0.6$ & 7 \\
\hline$<=0.6$ and $>0.4$ & 5 \\
\hline$<=0.4$ and $>0.2$ & 3 \\
\hline$<=0.2$ & 1 \\
\hline
\end{tabular}

Finally, we state the algorithm of the proposed protocol.

\section{E. Algorithm for the proposed protocol: algorithm of the proposed Adaptive WLB-AODV protocol}

Step 0: Use the WLB-AODV routing protocol for routing packets within the MANET.

Step 1: Initialize the first TTL value. Let us call it TTLO.

Step 2: Determine the farthest active source using the maximal source coverage algorithm. Set TTLn to the newly calculated value.

Step 3: Set the TTL value of the next cycle of gateway advertisement messages to the TTLn.

Step 4: Calculate the gateway advertisement interval periodicity for the next cycle using Table II. Step 5: Repeat steps 2 to 4.

\section{Gateway Selection Mechanism :}

As mentioned in the previous section, to select an appropriate gateway, we need to calculate the QoS metrics of each candidate gateway. Simple additive weighting method is used to combine these three QoS metrics as one comparable metric. The advantage of this decision making technique is that it considers multiple criteria, such as weight of importance level and gateway outranking according to the user preference or priority. So, this technique is effective to solve the quality ranking of these candidate gateways based on these three QoS metrics. Since the hop count metric can be achieved in the GWADV message sent by the gateway, here we define the method for estimating the current traffic load of the candidate gateway and path quality between the MANET node and the gateway.

1) Traffic load of gateway: The traffic load of gateway is defined as the average queue length of the network layer interface of the gateway in the MANET. To distribute the traffic load evenly over multiple gateways instead of overloading a few gateways, we use (1) to estimate the current

traffic load of gateway $\mathrm{i}$ at time $\mathrm{T}$ :

$\mathrm{Q}(\mathrm{i}, \mathrm{T})=\mathrm{aXQ}(\mathrm{i}, \mathrm{T}-2 \square \mathrm{T})+\mathrm{jJxQ}(\mathrm{i}, \mathrm{T}-\square \mathrm{T})(1)$

Where $\mathrm{O} \square \mathrm{a}, \mathrm{f} 3 \square 1, \mathrm{a}+\mathrm{f} 3=\mathrm{l}, \mathrm{L} 1 \mathrm{~T}$ is an estimation window. Furthermore, we bring this metric value into non-dimensional value using (2) to calculate the combined metric conveniently.

$\mathrm{LB}(\mathrm{i})=\mathrm{Q}(\mathrm{i}, \mathrm{T})(2) \mathrm{Q} \_\max (\mathrm{i})$

Where $Q_{-} \max (i)$ is the maximum queue length of the network layer interface of gateway $i$ in MANET. According to (2), we can infer that the smaller the value of $\mathrm{LB}(\mathrm{i})$, the less congestion of the gateway. To disseminate the information of the traffic load of the gateway to the MANET nodes, we modify the format of the GWADV message and add a new field called gateway -'oad to record the status of the traffic load of the gateway.

2) Path Quality: To estimate the quality of wireless path between the MANET node and the gateway, we use the technique used in. The original MANET nodes periodically broadcast small probe packets. Based on the average success 
rate of these packets in either direction on the wireless link, an approximation of link quality can be made. The expected link quality is estimated using (3):

$E L Q(i)=11 \mathrm{~d} f(i)(3)$

Where df represents the forward packet delivery ratio of the probe packets. So the path quality can be calculated using (4):

$$
\text { Path Quality }(p)=\text { "IELQ(i) (4) IEp }
$$

According to (3), the higher the value of the forward packet delivery ratio of the probe packets, the smaller the value of ELQ(i). SO, according to (4), the smaller the value of the Path_Quality(p), the better the path quality between the MANET node and the gateway.

3) Hop count: it is defined as the number of hops from the MANET node to the gateway. After the mobile node gets the hop count and the gateway load and completes the calculation of the path quality, it then uses (5) to combine these three QoS metrics.

GS = axhop _ count + j3xLB(i) + rXPath__uality(P) (5)

Where $\mathrm{O} \square \mathrm{a}, 13, \mathrm{y} \square \mathrm{l}, \mathrm{a}+\mathrm{j} 3+\mathrm{y}=\mathrm{l}$, hop_count represents the hop count from the MANET node to the gateway.After obtaining the GS value of each candidate gateway, we can finally outrank them. The smallest one will be selected as a gateway.

\section{SIMULATION SETUP AND WORK ANALYSIS}

This section describes the Simulation Setup, Parameters, Performance Metrics ans Simulation results used in this study. Moreover, it presents the parameters used in the simulations.

\section{Simulation Setup:}

We have simulated two scenarios, one with 15 nodes and the other with 25 nodes with the aim of testing the proposed protocol in a normal sparse environment ( 15 nodes) and a dense environment ( 25 nodes). The common parameters for both these simulation scenarios are given in table 2 . For both the scenarios, the variable parameter was speed of mobile node which varies between $1 \mathrm{mt} / \mathrm{sec}$ to $6 \mathrm{mts} / \mathrm{sec}$.

\section{Parameters:}

The parameters for simulation scenario for simple wireless are given in table 3.

The transmission range is the maximum possible distance between two communicating mobile nodes. If the distance between two mobile nodes is larger than $250 \mathrm{~m}$ they cannot communicate with each other directly.

Table 3: General parameters used in all simulation for wireless

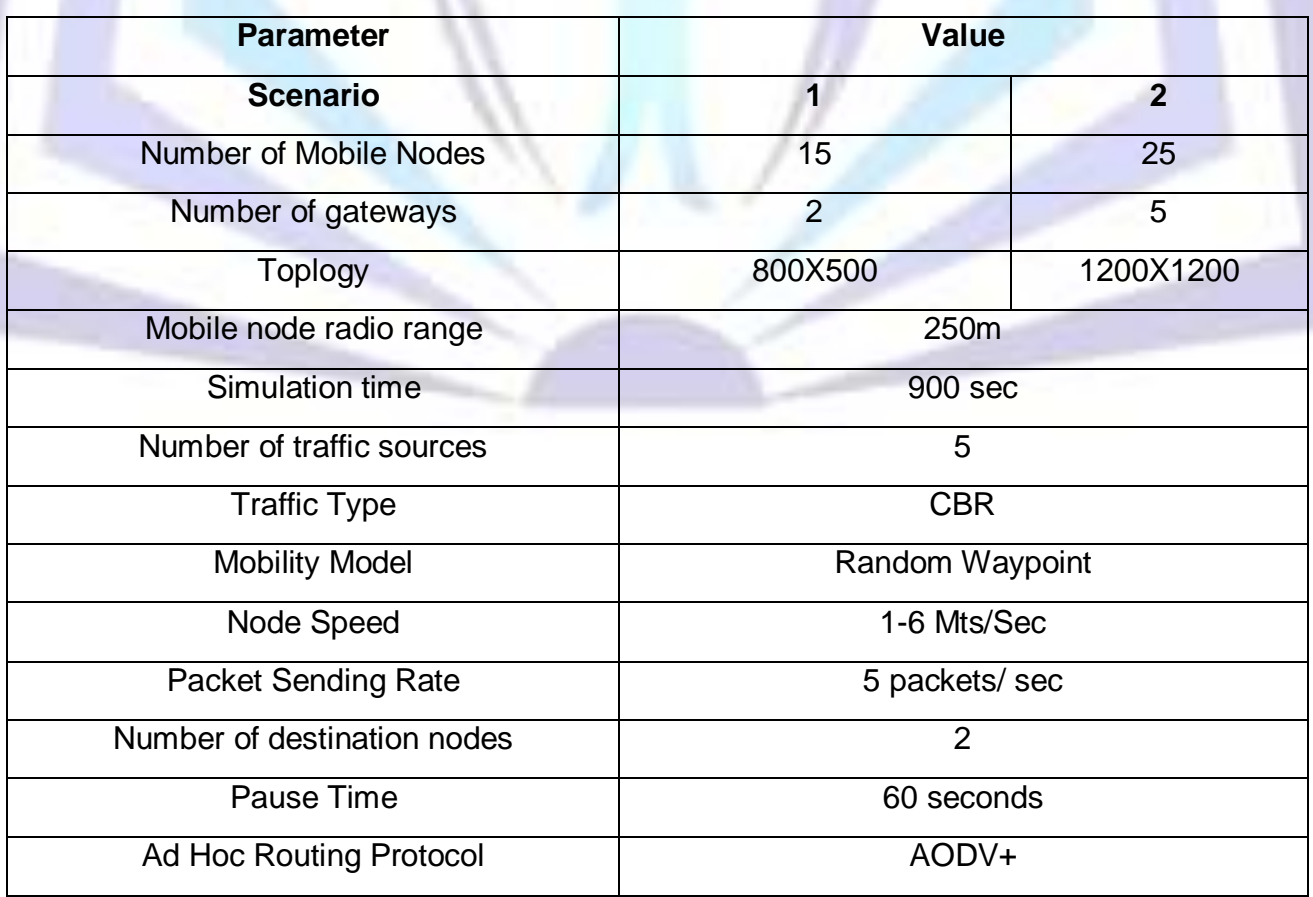




\section{Performance Metrics:}

The performance of the proposed routing protocol is analyzed with respect to the following performance metrics: Packet Delivery Ratio: It is defined as the percentage of the number of packets received by the destination to the total number of packets sent by the source. It is measured in terms of percentage.

End-to-End Delay: This is the average overall time taken (delay) for a packet to traverse from a source node to a destination node.

Normalized Routing Load: It is the ratio of the number of control packets generated to the number of data packets generated.

\section{Simulation Results:}

We have performed two separate sets of simulations for two different scenarios. For the first scenario, 15 mobile nodes were taken and for the second scenario, 25 mobile nodes were taken. We call these two scenarios, scenario-1 and scenario-2 respectively.

Packet delivery ratio: In Figures 5 and 6 we show the packet delivery ratio of scenario- 1 and scenario-2. We observe that the proposed protocol give better packet delivery ratio than the existing protocol for the entire node speeds in both the scenarios. Thus, we conclude that the proposed protocol outperforms the existing protocol in terms of packet delivery ratio for both sparse and dense environments.

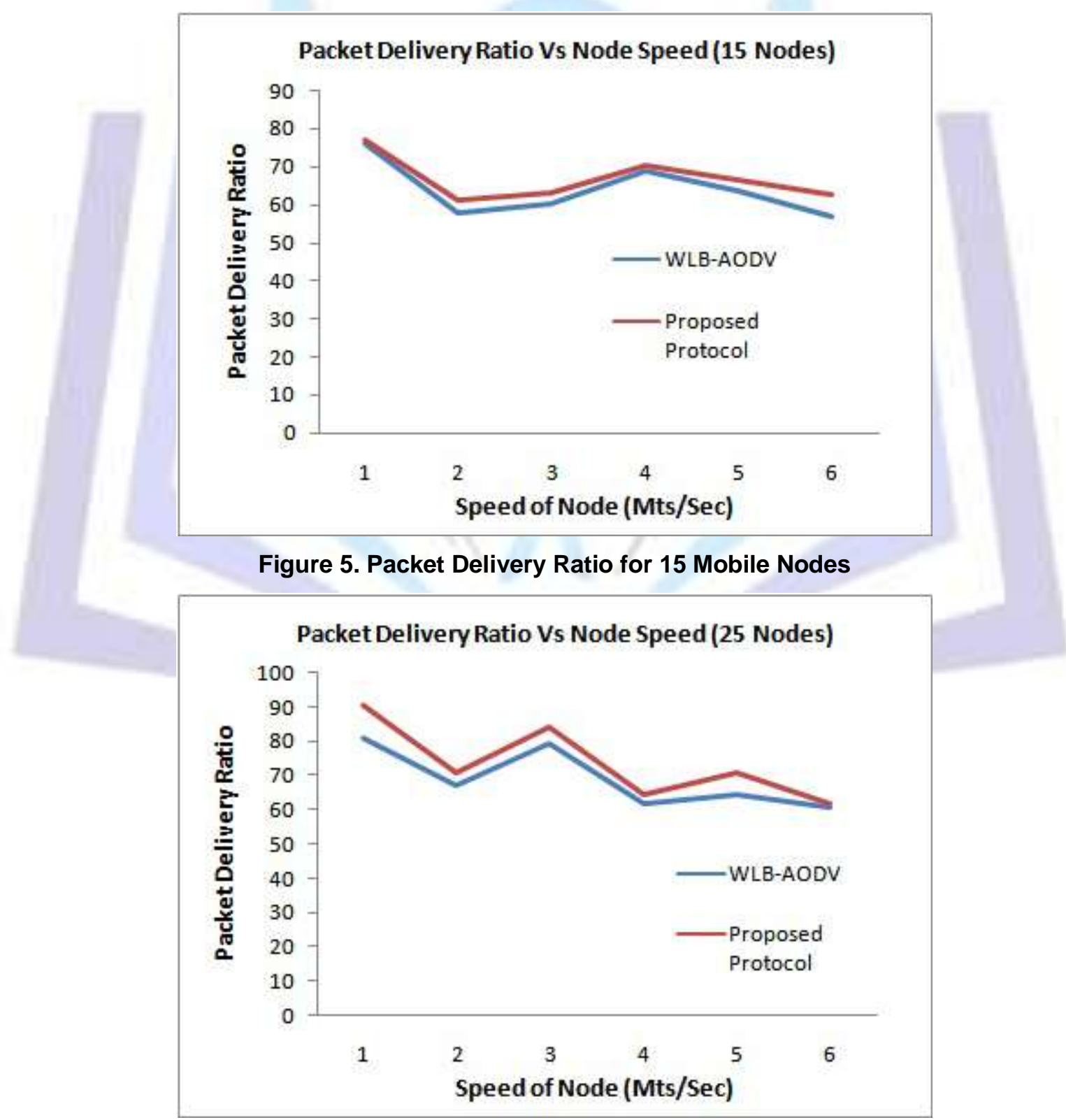

Figure 6. Packet Delivery Ratio for 25 Mobile Nodes 
End to end delay: In figures 7 and 8, we show the end to end delay performance comparison. We observe that the proposed protocol performs better than the existing protocol for scenario-1. The existing protocol gives better performance when it comes to scenario-2. Thus we conclude that the proposed protocol performs better in sparse environments and the existing protocol performs better in dense environments in terms of end to end delay. The delay may be more due the extra computation required at each node in the proposed protocol.

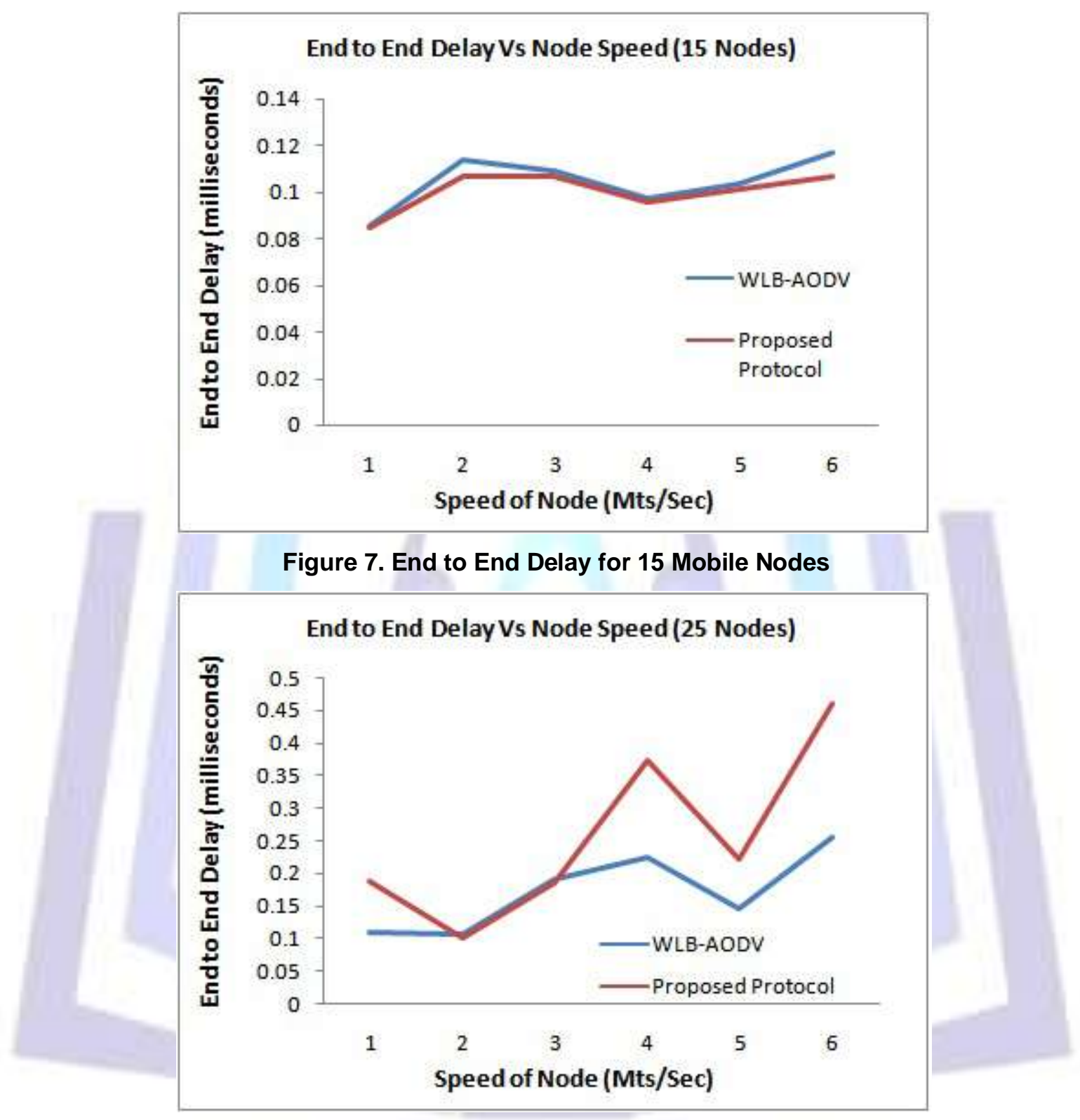

Figure 8. End to End Delay for 25 Mobile Nodes

\section{CONCLUSION \& FUTURE SCOPE}

It is evident from the graphs that there is a significant difference in the QoS of WiFi Network with Internet Discovery Gateway and without the Internet Discovery Gateway. In this work two parameters of QoS have been compared. This work can be extended to show the results of packet loss. Moreover in this paper only the WiFi network has been considered but in future simulation studies can be performed to study other networks like WiMax and WSN.

\section{REFERENCES}

[1] Shuo Ding, "A survey on integrating MANETs with the Internet:Challenges and designs", Computer Communications, vol 31 issue 14, pp 3537-3551, (2008)

[2] C.E. Perkins, "Mobile Networking Through Mobile IP," IEEE Internet Computing, Vol. 2, Issue 1, ( 1998)

[3] Khaleel Ur Rahman Khan, Rafi U Zaman, A.Venugopal Reddy, "Integrating Mobile Ad Hoc Networks and the Internet: challenges and a review of strategies", Communication Systems Software and Middleware and Workshops, COMSWARE 2008. 3rd International Conference, 536 -543, (2008). 
[4] J.H. Zhao, X.Z.Yang and H.W.Liu, "Load-balancing Strategy of Multigateway for Ad hoc Internet Connectivity", Proceedings of the International Conference on Information Technology: Coding and Computing (ITCC'05) - Volume II, Pages: 592 - 596, (2005).

[5] A. Trivino-Cabrera, Eduardo Casilari, D. Bartolome and A. Ariza,"Traffic Load Distribution in Ad Hoc Networks through Mobile Internet Gateways", Proceedings of Fourth International Working Conference on Performance Modelling and Evaluation of Heterogeneous Networks, (2006).

[6] Y-Y Hsu, Y-C Tseng, C-C Tseng, C-F Huang, J-H Fan and H-L Wu, Design and Implementation of Two-Tier Mobile Ad Hoc Networks with Seamless Roaming and Load-Balancing Routing Capability", Proceedings of the First International Conference on Quality of Service in Heterogeneous Wired/Wireless Networks, Pages: 52 - 58, (2004).

[7] Rafi U Zaman, Khaleel Ur Rahman Khan, A. Venugopal Reddy, "A Review of Gateway Load Balancing Strategies in Integrated Internet- MANET", IEEE International Conference on Internet Multimedia Services Architecture and Applications (IMSAA), 1-6 (2009).

[8] Pedro M. Ruiz, Antonio F. Gomez-Skarmeta: "Maximal Source Coverage Adaptive Gateway Discovery for Hybrid Ad Hoc Networks", Lecture Notes in Computer Science, vol.3158, 28-41, (2004).

[9] A.J. Yuste, Alicia Trivino, F.D. Trujillo, E. Casilari: "Using Fuzzy Logic in Hybrid Multihop Wireless Networks". International Journal of Wireless \& Mobile Networks Volume 2, Issue 3, 96-108, (2010).

[10] Javaid, U., Rasheed, T.M., Meddour, D., Ahmed, T.: "Adaptive Distributed Gateway Discovery in Hybrid Wireless Networks". WCNC - 2008, 2735-2740. (2008).

[11] Zaman, Rafi U., and A. Venugopal Reddy. "A Survey of Adaptive Gateway Discovery Mechanisms in Heterogeneous Networks." International Journal of Computer Network and Information Security (IJCNIS) 5, no. 7 (2013): 34.

[12] Zaman, Rafi U., Khaleel-Ur-Rahman Khan, and A. Venugopal Reddy."Gateway load balancing in integrated internetMANET using WLBAODV."InProceedings of the International Conference and Workshop on Emerging Trends in Technology, pp. 411-416. ACM, (2010).

[13] A.J. Yuste, Alicia Trivino, F.D. Trujillo, E. Casilari: "Using Fuzzy Logic in Hybrid Multihop Wireless Networks". International Journal of Wireless \& Mobile Networks Volume 2, Issue 3, 96-108, (2010).

[14] Zaman, R.U.; Khan, K.U.R.; Venugopal Reddy, A., "Mamdani Fuzzy Control based adaptive gateway discovery for ubiquitous Internet access in MANET," India Conference (INDICON), 2014 Annual IEEE , vol., no., pp.1,5, 11-13 Dec. 2014

[15] Zaman, R.U.; Khan, K.U.R.; Venugopal Reddy, A., "Load balanced fuzzy control based adaptive gateway discovery in Integrated Internet MANET," International Conference on Computer and Communications Technologies (ICCCT), 2014, vol., no., pp.1,6, 11-13 Dec. 2014.

[16] Yonghang Yan; Linlin $\mathrm{Ci}$; Zhiming Wang; Wei He, "QoS-based gateway selection in MANET with Internet connectivity," 15th International Conference on Advanced Communication Technology (ICACT), 2013 , vol., no., pp.195,199, 27-30 Jan. 2013. 